\title{
Human Identification via Face Recognition: Comparative Study
}

\author{
${ }^{1}$ Associate Professor, Dept. of Computer Science \&Engg., AcharyaNagrjuna University, India \\ [satayaprasad ravi@gmail.com] \\ ${ }^{2}$ University of Human Development, Department of Computer Science, Sulaimani, Iraq \\ [muzhir.al-ani@uhd.edi.iq] \\ ${ }^{3}$ AcharyaNagarjunaUniversity, Department of Computer Science \& Engineering, India \\ [salwa12005@yahoo.com]
}

\begin{abstract}
Biometric recognition becomes an attractive issue of processing, and have a vast amount of real applications.According to wide spread of Internet and the new aspect of Internet of things, millions of human characteristics are flying around the world. So the security and identification become a big challenge and most of the individual needs to apply secure system to save their information. Face recognition is a narrow field of biometric recognition but there are vast amount of works and projects concerned this field. These works used various technique to reach high accuracy of recognition. The big challenge of face recognition is how to improve the recognition rate according to the implemented system. The main aim of this approach is concern with the scientific survey of the last two years to generate a simple comparative study of techniques, methods, challenges and benefits of applying these approaches.Mean Square Error (MSE) and Peak Signal to Noise Ratio (PSNR) are measured for all the tested methods $2 D$-WHT, 2D-DCT, 2D-DWT, 2D-FFT and M-2D-DWT in which the results obtained from M-2D-DWT gives better values of MSE and PSNR.
\end{abstract}

Keywords: Biometrics; Face Recognition; Human Identification; Security.

\section{Introduction}

Now adays human identification via face recognition methods are well known issues and have a wide range of applications [1],[2].Face recognition is an attractive filed that combining of image processing, statistical measures, pattern recognition and computer vision and other related fields [3],[4],[5].Different methods of face recognition are designed and implemented such as discrete wavelet transform [6], Curvelet Transform [7], discrete cosine transform [8], principal component analysis [9], independent component analysis [10].Face recognition is an important field of Image processing, and it play an important part of security field [11],[12],[13].Face image representation using different techniques are applied [14],[15].Image Fusion methods for classification and extraction are used to reconstruct the image [16],[17],[18].Fusion technique can be applied for images and videos [19],[20].Quality improving of image is a good issue and it is required to reduce the error as possible and reaching high accuracy [21],[22],[23].Face recognition and enhancement are implemented via different methods [24],[25].Image watermarking leads to security aspect of face identification [26],[27],[28].After this brief introduction we can say that there are many techniques (DWT, DFT, DCT, PCA, SVM ...etc.) used for face recognition and the accuracy of these technique depends of many factors such as type of data set, focusing, lighting, resolution, ... etc. This work will concentrated on the well-known techniques, in addition some focusing goes to the benefits of these techniques.

\section{Literature Review}

Big amount of papers are published in this field, and in order to resize our challenge, we will concentrated in this section about the paper published in the last two years.Li Li, JianqiangGaob, HongweiGea (2016) investigated a semi-discrete decomposition method to solve sample imaging problems. Firstly, applying semi-discrete decomposition on original image and its translation via the training set. Next, applying scatter matrices on original image and two approximation images. Then, fined the optimal projection vectors via applying the proposed algorithm.In addition, applying the final classification usingthe closest neighbor classifier. The performance of the proposed method is evaluated on the basis of ORL, Yale and FERET data. The column rotation is implemented to perform the recognition rate applied via experimental test [29].

Hongjun Li, Ching Y. Suen (2016), considered the problem of recognizing the human face from frontal views with variable illumination and occlusion and concealment. They presented an efficient approach face recognition algorithm using extracting dynamic images. Parts of images are used to represent the typical discriminative components that are implemented to classify face images. The experimental results achieved highaccuracy, robustness and speed [30].Biao Leng et al. (2016), proposeda new method to generate reasonable virtual samplesin order to prevent imbalance in classification operation. This method developed boost approach andused face analysis based on Joint Bayesian. This approach achieve an effective experimental results for feature extraction using conventional neural networks [31]. 
Daniel B. Elbich and K. Suzanne Scherf (2016), implemented the individual differences in brainbehavior according to face recognition system for both core and extended regions. Hybrid measures of behavioral and neural are applied to assessbrain behavior relations. This approach measures and evaluateface and object recognition behaviorfor each region, in addition to the global activation of regions. An effective results are obtained via applying this approach [32].

ShonalChaudhry and Rohitash Chandra (2016), introduced a mobile face detection and recognition approach when the data is acquisition from mobile source. This approach is applied using convolutional neural networks. A video database is created via capturing videos from camera of mobile that faced with many problems via the application. A good results are obtained via applying for daylight and artificial lighting conditions while the other conditions are not acceptable. This approach can be applied for other devices such as smartphones and wearable devices [33].

Xiao Dong (2017), designed face recognition approach based on collaborative representation techniques. This approach is applied via two stage learning methodof face recognition. Thecollaborative coefficients are obtained firstly using unlabeled samples to construct the labeled samples. In order to label all samples in the database, this process is repeated until the operation is completed. The obtained results indicated that this approach is an effective in face recognition [34].

G. Besson (2017), explained the comparison between three approaches of face recognition. These methods are face recognition via face familiarity, superordinate face recognitionand target face person.This approach is implemented for 27 individuals. The approach applied via recent speed and accuracy boosting procedure in order to use their fastest strategy. The high speed is achieved in this approach via applying face recognition for finding a specific person in a crowd location, this lead only a quarter of a second [35].

Q. Wang et al. (2017), optimizedthe ability of composite filters for noise reduction. This approach extract the features and boundaries of the training faces. The composite correlation filter that applied here have a simple implementation approach and does not have any mathematical complexity. A simulation test is applied to ensurethe effectiveness and feasibility of the system. The true positive and false positive rates are measured in this system in an efficient way [36].

Hengmin Zhang et al. (2017), described an efficient approach of robust face recognition. This paper focusing on that the existing face recognition and classification methods concentrated on characterize the representation error.This approach is implemented using nuclear norm to describe the low- rank structural information, on the other hand this may leads to suboptimal solution. This approach leads to an optimal results [37].C-H.Yoo et al. (2017), improved an effective feature extraction method for classifying images. This method realizeand improve the ability for face recognition. This approach is implemented via the partition of thelocal binary pattern into bit planes that specified the face image direction. Then complaining all bit planes to generate the feature vector. The experimental results showed that this method achieve high performance compared with the existing methods [38].

\subsection{Adapted Image Data}

\section{Methodology}

In order to be fair for the existing method for human identification via face recognition, we apply each approach on the same set of data.In order to reach the identical case of realization, The Database of Faces that used here is "The ORL Database of Faces" that have 400 faces of 10 different situations for 40 persons. The image size of this database is $92 * 112$ pixels. So the first step to adapt these image into Matlab package, these images are resized into $128 * 128$ pixels to be adapted for fast transforms as shown in figure 1 .

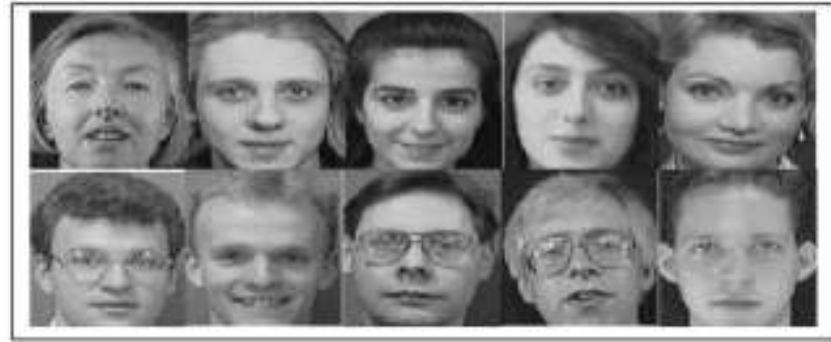

Figure 1 resized images into $128 * 128$ pixels

\subsection{Implemented Approach}

Matlab environment is used to implement various techniques of face recognition. It is impossible to cover all techniques used in this field. So we will concentrated on the well-known techniques, and which have a wide range of applications. Five methods (D-WHT, 2D-DCT, 2D-DWT, 2D-FFT and M-2D-DWT) are explained below. 
Two Dimensional Fast Fourier Transform (2D-FFT) that is the fast version of 2D-DFT. The application of this technique on images leads to real part an imaginary part. This technique can be implemented via direct convolution in time domain or indirect convolution in frequency domain. There are many types of FFT and these types depends of the radix of the input data and the most important types are FFT radix2 (base 2) and FFT radix4 (base 4). This technique have a wide range of applications in image processing including face recognition.

Walsh Hadmard Transform (2D-WHT) The fast Walsh-Hadamardtranform algorithm is similar to the Cooley-Tukey algorithm used for the FFT. Both use a butterfly structure to determine the transform coefficients. Two Dimensional Discrete Cosine Transform (2D-DCT) is an interested technique used for many purposes including face recognition. The output of 2D-DCT (when applied to input image) is only real part. In 2DDCT, the input image is divided into 8 -by-8 or 16-by-16 blocks, and the two-dimensional DCT is computed for each block. The DCT coefficients are then quantized, coded, and transmitted.

Two Dimensional Discrete Wavelet Transform (2D-DWT) is a very famous technique used for face recognition. There are various types of masks or families are used to implement 2D-DWT. These masks are Harr, Daubechies, Morlet, Meyer, Mallat ... etc. All these masks have the similar procedure for implementation bus they have various weights. The architecture of this technique depends on siplitting the output into four main components. The low-low (LL) component are generated via applying low pass filter on rows of the input image to get low band then apply low pass filter on columns of the output of low band to get LL component. The low-high (LH) component are generated via applying low pass filter on rows of the input image to get low band then apply high pass filter on columns of the output of low band to get LH component. The high-low (HL) component are generated via applying high pass filter on rows of the input image to get low band then apply low pass filter on columns of the output of low band to get HL component. The high-high (HH) component are generated via applying high pass filter on rows of the input image to get high band then apply high pass filter on columns of the output of high band to get HH component.

Modified Two dimensional discrete wavelet transform (M-2D-DWT) is generated via modifying the weighting factor of 2D-DWT. Weighting factor of 2D-DWT can takes different values smaller than one and greater than one. According to the testing of different values of weighting factor, we reach a reasonable value that serve for the comparison.

\section{Results And Analysis}

The experiment was started by implementing the methods and functions under Matlab package. These methods and functions are directly related to face recognition. These functions are 2D-WHT, 2D-DCT, 2DDWT, 2D-FFT and M-2D-DWT. All these functions are applied to the adapted ten images (five male and five female) of size $128 * 128$ pixels. These images start with the number image $11 \mathrm{~m}$ and end with the number $20 \mathrm{f}$.Let the original image is $\mathrm{x} 1(128 * 128$ pixels) and the retrieved image is $\mathrm{x} 2(128 * 128$ pixels) then we measured Mean Square Error (MSE) and Peak Signal to Noise Ratio (PSNR) for all the tested methods 2D-WHT, 2DDCT, 2D-DWT, 2D-FFT and M-2D-DWT as shown in the following two equations.

$M S E=\sum \sum \sum \frac{(x 1-x 2)^{2}}{128 * 128}$
$P S N R=10 * \log 10\left(\frac{128 * 128}{M S E}\right)$

Table 1 illustrate MSE values between the original images and the retrieved images, in which the obtained results of the methods 2D-DWT, 2D-FFT and M-2D-DWT are the best results.In addition the MSE obtained from M-2D-DWT is the best one compared with the others. These results also are clear in figure 2 which demonstrates the MSE of both 2D-FFT and M-2D-DWT only because the other methods are out of range. This figure indicates a big difference between 2D-FFT and M-2D-DWT, means M-2D-DWT deals with better performance.

Table 1 MSE for all tested methods

\begin{tabular}{|l|l|l|l|l|l|}
\hline MSE & 2D-WHT & 2D-DCT & 2D-DWT & 2D-FFT & M-2D-DWT \\
\hline Image11m & $1.9486 \mathrm{e}+004$ & 18.0954 & $1.1058 \mathrm{e}-021$ & $1.6939 \mathrm{e}-027$ & $1.9611 \mathrm{e}-028$ \\
\hline Image12m & $1.5578 \mathrm{e}+004$ & 16.6347 & $1.6768 \mathrm{e}-021$ & $1.4411 \mathrm{e}-027$ & $1.2204 \mathrm{e}-028$ \\
\hline Image13m & $1.5340 \mathrm{e}+004$ & 16.8169 & $1.4509 \mathrm{e}-021$ & $1.5628 \mathrm{e}-027$ & $1.4064 \mathrm{e}-028$ \\
\hline Image14m & $1.7930 \mathrm{e}+004$ & 19.2712 & $6.9203 \mathrm{e}-022$ & $1.7330 \mathrm{e}-027$ & $4.8440 \mathrm{e}-029$ \\
\hline Image15m & $1.8119 \mathrm{e}+004$ & 17.4226 & $1.2278 \mathrm{e}-021$ & $1.7372 \mathrm{e}-027$ & $9.2898 \mathrm{e}-029$ \\
\hline Image16f & $1.6823 \mathrm{e}+004$ & 18.7300 & $7.9118 \mathrm{e}-022$ & $1.9173 \mathrm{e}-027$ & $2.2315 \mathrm{e}-028$ \\
\hline Image17f & $1.2744 \mathrm{e}+004$ & 17.9742 & $1.1694 \mathrm{e}-021$ & $1.2124 \mathrm{e}-027$ & $2.1466 \mathrm{e}-028$ \\
\hline Image18f & $2.0139 \mathrm{e}+004$ & 18.1617 & $1.1754 \mathrm{e}-021$ & $2.0495 \mathrm{e}-027$ & $1.3507 \mathrm{e}-028$ \\
\hline Image19f & $1.7212 \mathrm{e}+004$ & 16.1245 & $1.8352 \mathrm{e}-021$ & $1.6471 \mathrm{e}-027$ & $1.1117 \mathrm{e}-028$ \\
\hline Image20f & $1.7625 \mathrm{e}+004$ & 18.0773 & $1.1458 \mathrm{e}-021$ & $1.7296 \mathrm{e}-027$ & $8.7770 \mathrm{e}-029$ \\
\hline
\end{tabular}




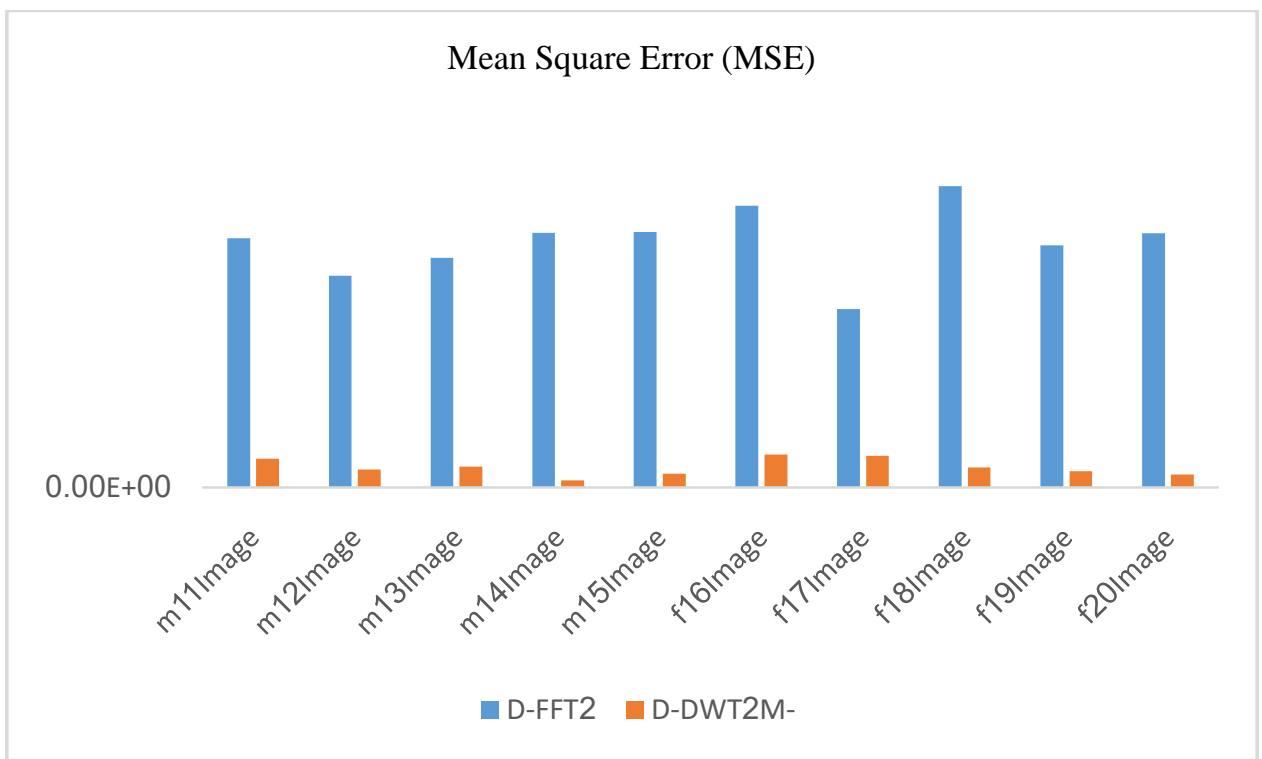

Figure 2 MSE for all tested methods

Table 2 illustrate PSNR values between the original images and the retrieved images of the methods 2D-WHT, 2D-DCT, 2D-DWT, 2D-FFT and M-2D-DWT in which the results obtained from M-2D-DWT are the best results. It is clear that the results obtained from the proposed method M-2D-DWT leads to be better than the other method. In addition these results are also demonstrated in figure 3. From this figure you can see that the three methods 2D-DWT, 2D-FFT and M-2D-DWT demonstrated near values of PSNR, means good results, and the best one mentioned is M-2D-DWT.

Table 2 PSNR for all tested methods

\begin{tabular}{|l|l|l|l|l|l|}
\hline PSNR & 2D-WHT & 2D-DCT & 2D-DWT & 2D-FFT & M_2D_DWT \\
\hline Image11m & 5.2335 & 35.5551 & 257.6939 & 315.8419 & 325.2058 \\
\hline Image12m & 6.2057 & 35.9207 & 255.8860 & 316.5439 & 327.2657 \\
\hline Image13m & 6.2725 & 35.8734 & 256.5144 & 316.1918 & 326.6499 \\
\hline Image14m & 5.5950 & 35.2817 & 259.7296 & 315.7427 & 331.2787 \\
\hline Image15m & 5.5496 & 35.7197 & 257.2395 & 315.7322 & 328.4508 \\
\hline Image16f & 5.8718 & 35.4054 & 259.1480 & 315.3040 & 324.6449 \\
\hline Image17f & 7.0778 & 35.5843 & 257.4512 & 317.2944 & 324.8134 \\
\hline Image18f & 5.0904 & 35.5392 & 257.4291 & 315.0142 & 326.8251 \\
\hline Image19f & 5.7725 & 36.0559 & 255.4939 & 315.9635 & 327.6708 \\
\hline Image20f & 5.6694 & 35.5595 & 257.5398 & 315.7514 & 328.6973 \\
\hline
\end{tabular}

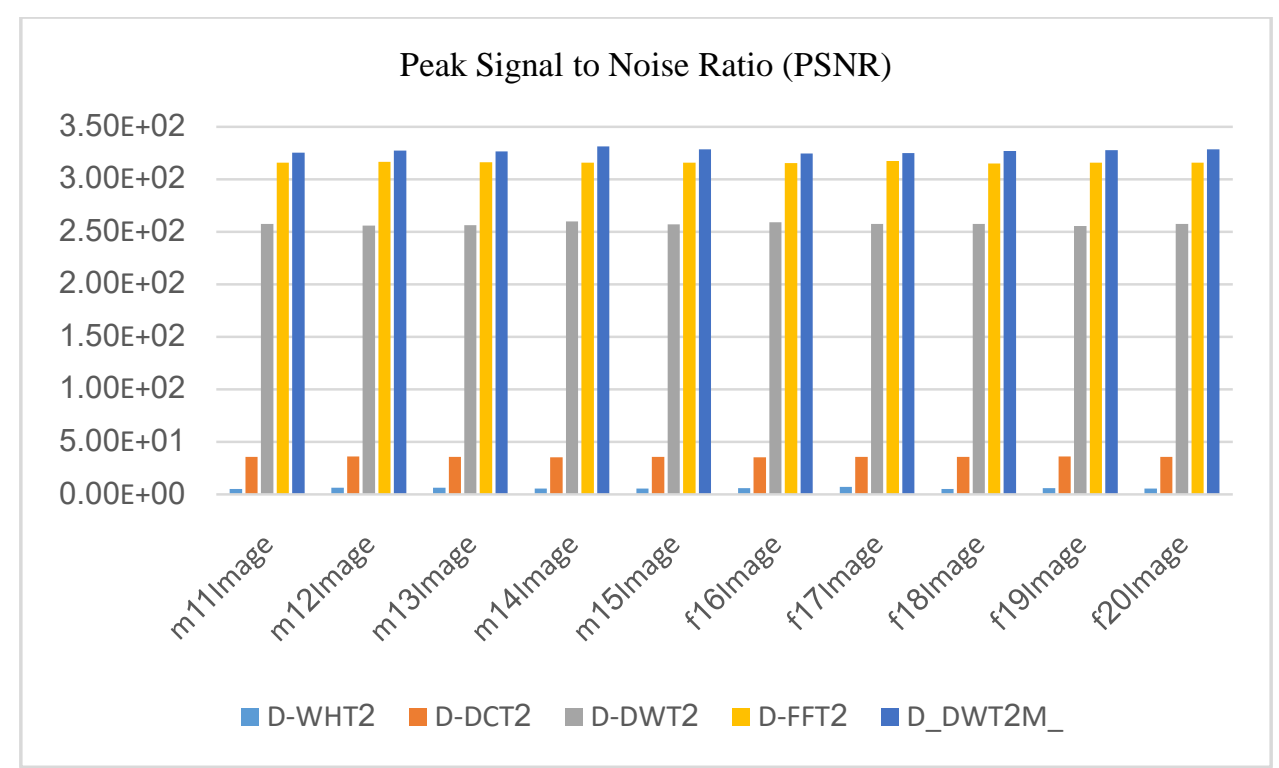

Figure 3 PSNR for all tested methods 


\section{Conclusions}

Face recognition is an important issue that used in human identification and introduced in huge amount of applications. Many face recognition methods (2D-WHT, 2D-DCT, 2D-DWT, 2D-FFT and M-2D-DWT) are implemented to compare their characteristics. To compare the performance of these methods, a database set of images are adapted and resized to fit with the implemented methods. MSE and PSNR are measured to compare the characteristics of the implemented methods for ten adapted tested images. The lower value of MSE means the lower of the error. The higher value of PSNR means the better quality of the compressed image, or reconstructed image. The obtained results indicated a good performance for both MSE and PSNR for the proposed M-2D-DWT method.

\section{References}

[1]. A. Wagner, J. Wright, A. Ganesh, Z. Zhou, H. Mobahi, Y. Ma, Toward a practical face recognition system: robust alignment and illumination by sparse representation, IEEE Trans. Pattern Anal. Mach. Intell. 34(2) (2012) 372-386.

[2]. L. Zhang, M. Yang, X. Feng, Sparse representation or collaborative representation: which helps face recognition?, in: ICCV, 2011.

[3]. Y. Xu, Q. Zhu, A simple and fast representation-based face recognition method, Neural Comput. Appl. 22(7-8) (2013) 1543-1549.

[4]. H. Zhang, Z. Zhang, Z. Li, Y. Chen, J. Shi, Improving representation-based classification for robust face recognition, J. Mod. Opt. 61(11) (2014) 961-968.

[5]. R. Satya Prasad, MuzhirShaban Al-Ani and Salwa Mohammed Nejres, "An Efficient Approach for Fingerprint Recognition", International Journal of Engineering Innovation \& Research, Vol. 4, Issue 2, 2015, India.

[6]. Ghazi IbrahemRaho, Ali JbaeerDawood and MuzhirShaban Al-Ani, "Real Time Fast Algorithm of 2D DWT Based DSP Technology", International Journal of Application or Innovation in Engineering \& Management, Volume 2, Issue 10, October 2013.

[7]. MuzhirShaban Al-Ani and AbdulrahmanDiraKhalaf , "Image Information Retrieval Using Wavelet and Curvelet Transform", International Journal of Soft Computing and Engineering (IJSCE) ISSN: 2231-2307, Volume-3, Issue-1, March 2013

[8]. MuzhirShaban Al-Ani and Wasan M. Al-Aloosi, "Biometrics Fingerprint Recognition using Discrete Cosine Transform (DCT)", International Journal of Computer Applications (0975 - 8887), Volume 69- No.6, May 2013.

[9]. K. Tan, S. Chen, Adaptively weighted sub-pattern PCA for face recognition, Neurocomputing 64 (2005) 505-511.

[10]. J. Yang, D. Zhang, A. Frangi, J.Y. Yang, Two-dimensional PCA: a new approach to appearance-based face representation and recognition, IEEE Trans. Pattern Anal. Mach. Intell. 26(1) (2004) 131-137.

[11]. Y. Xu, Z. Jin, Down-sampling face images and low-resolution face recognition, in: Proceedings of 3rd Int. Conf. Innovative Computing, Information and Control, 2008, pp.392-395.

[12]. Y. Xu, Q. Zhu, Y. Chen, J. Pan, An improvement to the nearest neighbor classifier and face recognition experiments, Int. J. Innovative Computing, Information and Control 9(2) (2013) 543-554.

[13]. Dr. R. Satya Prasad, MuzhirShaban Al-Ani, Salwa Mohammed Nejres, "An Efficient Approach for Human Face Recognition", International Journal of Advanced Research in Computer Science and Software Engineering, IF=2.5, Volume 5, Issue 9, September 2015, India.

[14]. C. Zhang, Combination of direct matching and collaborative representation for face recognition, J. Mod. Opt. 60(10) (2013) 831835 .

[15]. R. Rubinstein, A.M. Bruckstein, M. Elad, Dictionaries for sparse representation modeling, Proc. IEEE 98(6) (2010) 1045-1057.

[16]. ] G. Gao, L. Zhang, J. Yang, L. Zhang, D. Zhang, Reconstruction based finger knuckle-print verification with score level adaptive binary fusion, IEEE Trans. Image Process. 22(12) (2013) 5050-5062.

[17]. S. Yang, W. Zuo, L. Liu, Y. Li, D. Zhang, Adaptive Weighted Fusion of Local Kernel Classifiers for Effective Pattern Classification, ICIC, 2011, pp.63-70.

[18]. Dr. R. Satya Prasad, MuzhirShaban Al-Ani, Salwa Mohammed Nejres, "Hybrid Fusion of Two Human Biometric Features", International Journal of Business and ICT, Volume 2 Number 1-2, June 2016.

[19]. Y. Xu, Y. Lu, Adaptive weighted fusion: a novel fusion approach for image classification, Neurocomputing 168 (2015) 566-574.

[20]. S. Kim, J.Y. Choi, S. Han, M.R. Yong, Adaptive weighted fusion with new spatial and temporal fingerprints for improved video copy detection, Signal Process. Image Commun. 29(7) (2014) 788-806.

[21]. W.Zhao, R.Chellppa, P.J.Phillips, A.Rosenfeld, Face recognition: a literature survey, ACM Comput. Surv. 35(4) (2003) 399-458.

[22]. Y.Q.Guo, R.He, W.S.Zheng, X.W.Kong, Z.F.He, Robust spectral regression for face recognition, Neurocomputing 118 (2013) $33-$ 40.

[23]. R. He, W.S.Zheng, B.G.Hu, X.W.Kong, Two-stage non negative sparse representation for large scale face recognition, IEEETrans. Neural Netw. Learn. Syst. 24(1)(2013)35-46.

[24]. W.R. Boukabou, A. Bouridane, S. Al-Maadeed, Enhancing face recognition using directional filter banks, Digit. Signal Process. 23(2) (2013) 586-594.

[25]. Z. Zhang, Y. Xu, J. Yang, X. Li, D. Zhang, A survey of sparse representation: algorithms and applications, IEEE Access 3 (2015) 490-530.

[26]. C.C. Lin, C.C. Chang, Y.H. Chen, A Novel SVD-based watermarking scheme for protecting rightful ownership of digital images, J. Inf. Hiding Multimedia Signal Processing 5(2) (2014) 124-143.

[27]. A. Benhocine, L. Laouamer, L. Nana, A.C. Pascu, New images watermarking scheme based on singular value decomposition, J. Inf. Hiding Multimedia Signal Processing 4(1) (2013) 9-18.

[28]. MuzhirShaban Al-Ani and KhetamAbdAl_Baset, "Efficient Watermarking based on Robust Biometric Features", IRACST Engineering Science and Technology: An International Journal (ESTIJ), ISSN: 2250-3498, Vol.3, No.3, June 2013.

[29]. Li Li, JianqiangGaob, HongweiGea, "A new face recognition method via semi-discrete decomposition for one sample problem", Optik 127 (2016) 7408-7417.

[30]. Hongjun Li, Ching Y. Suen, Robust face recognition based on dynamic rank representation, Pattern Recognition, http://dx.doi.org/10.1016/j.patcog.2016.05.014, Accepted date: 10 May 2016.

[31]. Biao Leng, Kai Yu, Yu Liu and Jingyan QIN, Data Augmentation for Unbalanced Face Recognition Training Sets, Neurocomputing, http://dx.doi.org/10.1016/j.neucom.2016.12.013, Accepted date: 6 December 2016.

[32]. Daniel B. Elbich and K. Suzanne Scherf, Beyond the FFA: Brain-Behavior Correspondences in Face Recognition Abilities, NeuroImage, http://dx.doi.org/10.1016/j.neuroimage.2016.12.042, Accepted date: 15 December 2016. 
[33]. ShonalChaudhry and Rohitash Chandra, Face Detection and Recognition in an Unconstrained Environment for Mobile Visual Assistive System, <![CDATA[Applied Soft Computing Journal]]> (2016), http://dx.doi.org/10.1016/j.asoc.2016.12.035, Accepted date: $15-12-2016$.

[34]. Xiao Dong, Huaxiang Zhang, Jiande Sun, WenboWana, “A two-stage learning approach to face recognition”, J. Vis. Commun. Image R. 43 (2017) 21-29.

[35]. G. Besson, G. Barragan-Jason, S.J. Thorpe, M. Fabre-Thorpe, S. Puma, M. Ceccaldi, E.J. Barbeau, "From face processing to face recognition: Comparing three different processing levels", Cognition, 158 (2017) 33-43.

[36]. Q. Wang, M. Elbouz, A. Alfalou and C. Brosseau, "Designing a composite correlation filter based on iterative optimization of training images for distortion invariant face recognition", Optics and Lasers in Engineering 93 (2017) 100-108.

[37]. Hengmin Zhang, Jian Yang, JianchunXie, JianjunQian, Bob Zhang, "Weighted sparse coding regularized nonconvex matrix regression for robust face recognition", Information Sciences 394-395 (2017) 1-17.

[38]. C-H. Yoo, S-W. Kim, J-Y. Jung, and S-J. Ko, High-Dimensional Feature Extraction Using Bit-Plane Decomposition of Local Binary Patterns for Robust Face Recognition, J. Vis. Commun. Image R. (2017), doi: http://dx.doi.org/10.1016/j.jvcir.2017.02.009, Authors Accepted Date: 10 February 2017.

${ }^{1}$ Dr. R. Satya Prasad received Ph.D. degree in Computer Science in the faculty of Engineering in 2007 from

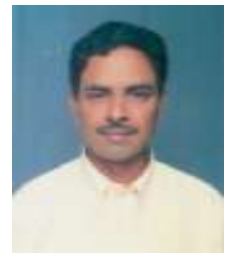
AcharyaNagarjuna University, Andhra Pradesh. He received gold medal from AcharyaNagarjuna University for his out standing performance in Masters Degree. He is currently working as Associate Professor and H.O.D, in the Department of Computer Science \& Engineering, AcharyaNagarjuna University. His current research is focused on Software Engineering, Software reliability. He has published several papers in National \& International Journals.

Authors

MuzhirShaban Al-Ani has received Ph. D. in Computer \& Communication Engineering Technology, ETSII,

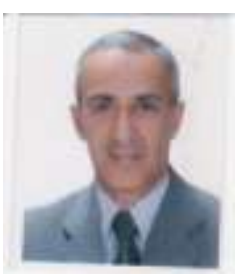
Valladolid University, Spain, 1994. Assistant of Dean at Al-Anbar Technical Institute (1985). Head of Electrical Department at Al-Anbar Technical Institute, Iraq (1985-1988), Head of Computer and Software Engineering Department at Al-Mustansyria University, Iraq (19972001), Dean of Computer Science (CS) \& Information System (IS) faculty at University of Technology, Iraq (2001-2003). He joined in 15 September 2003 Electrical and Computer Engineering Department, College of Engineering, Applied Science University, Amman, Jordan, as Associated Professor. He joined in 15 September 2005 Management Information System Department, Amman Arab University, Amman, Jordan, as Associated Professor, then he joined computer science department in 15 September 2008 at the same university. He joined in August 2009 College of Computer Science, Al-Anbar University, Al-Anbar, Iraq, as Professor.He joined in 1 October 2016 Department of Computer Science - College of Science and Technology - University of Human Development - KRG - Iraq.

\section{Authors}

${ }^{3}$ Salwa Mohammed Nejres has received in B.Sc. in Computer Science from University of Mustansiriya,

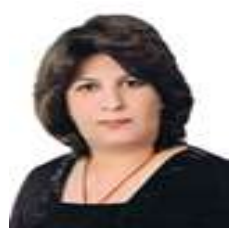
Department of Computer Sciences, Iraq (2001-2002). She completed her M.Sc. in Computer Science Department at AL- Anbar University in the field of data mining (2009-2010). She is currently a Ph.D. student in computer Science Department of AcharyaNagarjuna University (ANU),Nagarjunanagar, India, ANU College of Engineering \& Technology. 